\title{
Semaphorin-3A is a repulsive but attractive renal guidance cue to therapy
}

\author{
Friedrich C. Luft
}

Published online: 22 October 2014

(C) Springer-Verlag Berlin Heidelberg 2014

The semaphorins are a family of secreted or membraneassociated glycoproteins that have been grouped into eight classes based upon their structural elements and amino acid sequences. Semaphorin classes 1, 4, 5, 6, and 7 are membraneassociated, while classes 2 and 3 , as well as the viral semaphorins, are secreted. They are characterized structurally by a conserved $\sim 400$ amino acid Sema domain [1]. Semaphorins are classically described as collapsing factors and mediators of axon repulsion, although they may also act as context-dependent chemoattractants. Their functions were subsequently extended to regulation of the immune system as "immune semaphorins." These semaphorins are involved in almost all phases of both normal and pathological immune responses and participate in allergic diseases and autoimmune diseases. Semaphorin (Sema)-3A downregulates autoimmune responses by suppressing the over-activity of both T- and Bcell autoimmunity. Other semaphorins, such as Sema-4D and Sema-4A, are important in stimulating $\mathrm{T}$ and B cells, thus maintaining these immune responses [2]. Semaphorins are even important to renal development. Gain- and loss-offunction experiments demonstrated that Sema-3A is required for proper podocyte differentiation, a normal glomerular filtration barrier, and vascular patterning. For the latter activity, Sema-3A acts as a negative regulator of endothelial cell migration and survival. Sema-3A is also required for ureteric bud morphogenesis [3]. Sema-3A is even involved in the development of acute kidney injury. Experimentally inactivating the molecule appears to suppress ischemia-reperfusioninduced inflammation [4]. Thus, it comes as no surprise that

F. C. Luft $(\bowtie)$

Experimental and Clinical Research Center, a joint cooperation between the Max-Delbrück Center for Molecular Medicine and the Charité Medical Faculty, Berlin, Germany

e-mail: luft@charite.de
Sema-3A is found in urine and could be a biomarker for acute kidney injury [5-7].

In this issue of $\mathrm{J}$ Mol Med, Mohamed and colleagues extend the semaphorin story to a chronic renal disease, namely diabetic nephropathy [8]. The group found increased Sema$3 \mathrm{~A}$ expression in podocytes, distal tubular cells, and collecting duct cells not only in streptozotocin-induced diabetes but also in genetic diabetic mouse models. Urinary Sema-3A excretion was increased as albuminuria developed. The authors extended their observations to patients with diabetes and found increased Sema-3A urinary excretion in the patients as well. The investigators then inspected streptozotocin-treated Sema$3 A$ gene-deleted mice and found that the mice were resistant to diabetes-induced albuminuria despite similar blood sugar values as wild-type mice and equivalent changes in body weight. The renal histology also supported the conclusion that the Sema-3A gene-deleted mice were protected from diabetic nephropathy. Tumor necrosis factor (TNF), interleukin (IL)-1, IL-6, macrophage chemoattractant protein (MCP)-1, transforming growth factor (TGF)- $\beta 1$, and collagen markers were all reduced in the gene-deleted model as well. Similar data were generated by the administration of a Sema-3A inhibitory peptide. Mohamed et al. also observed that diabetes increased the expression of the Sema-3A receptors, neuropilin-1 and neuropilin-2. Finally, the authors observed that gene deletion or peptide inhibition of Sema-3A resulted in less oxidative stress in the diabetic kidneys as indicated by reduced thiobarbituric acid-reactive substances (TBARS) in urine. The findings are both impressive and compelling.

Sema-3A appeared in the context of diabetic complications before. Another major target organ in diabetes is the eye. Deterioration of the inner blood-retinal barrier and consequent macular edema are cardinal manifestations of diabetic retinopathy. As a matter of fact, macular edema is the clinical feature most closely associated with loss of sight. Cerani and colleagues provided evidence from both human and animal 
studies for the critical role of the Sema-3A protein in instigating pathological vascular permeability in diabetic retinas via the Sema-3A receptor, neuropilin-1 [9]. They also observed that Sema-3A was induced in early hyperglycemic phases of diabetes within the retina and precipitated an endothelial barrier-function breakdown. Cerani et al. showed that neutralization of Sema-3A signaling prevented diabetes-induced retinal vascular leakage in a stage of the disease when vascular endothelial growth factor neutralization was not efficient [9]. They then corroborated their observations in conditional neuropilin-1 gene-deleted mice. Their findings identified Sema-3A signaling as a possible therapeutic target for macular edema and diabetic retinopathy similar to what Mohamed and colleagues show for the kidney in their report [8].

How could Sema-3A contribute to diabetes-induced targetorgan damage? Mohamed et al. suggest that oxidative stress could be responsible, as their findings with increased TBARS in urine would suggest [8]. The Sema-3A protein and oxidative stress certainly appear to interact. Smith et al. have previously demonstrated in a model of oxygen-induced retinopathy that in late stages of pathological retinal neovascularization, neuronal-derived Sema-3A forms a repulsive barrier that hinders normal revascularization by misdirecting vessels away from the ischemic retina [10]. Suppression of Sema$3 \mathrm{~A}$ has also been suggested as a therapy for that form of retinopathy.

Furthermore, Sema-3A is said to increase toll-like receptor (TLR)-4 signaling that could foster nuclear factor kappaB (NF-KB) activation and inflammation. Wen et al. found that the administration of Sema-3A, which is both a neuropilin and

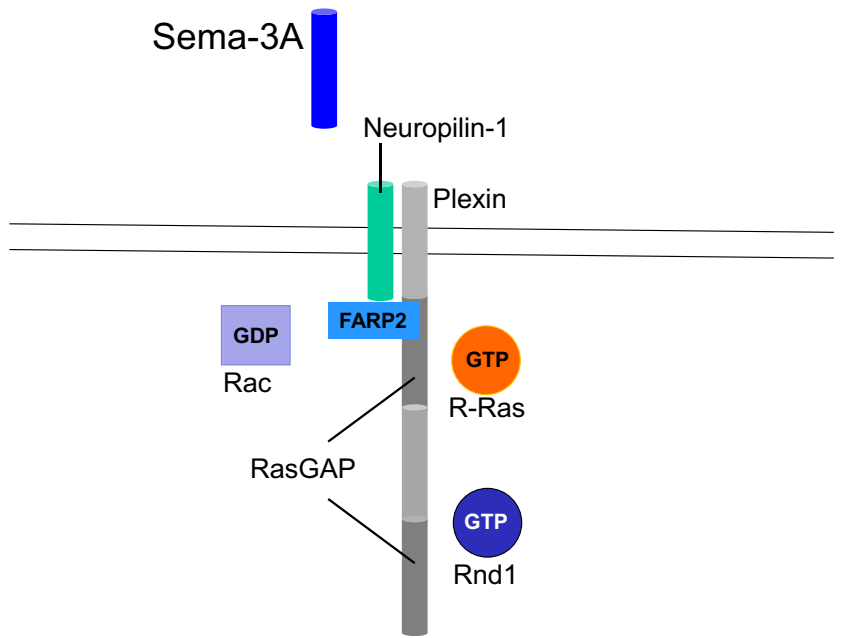

Fig. 1 Semaphorin-3A (Sema-3A) acts through a receptor complex containing the single-pass transmembrane proteins neuropilin, which binds Sema-3A, and plexin, which transduces the signal. Integrin inhibition and growth-cone repulsion responses to Sema-3A are dependent on the activities of small GTPases, including Rac and Rnd1. Also other events, such as apoptosis and possibly inflammatory pathways, depend on the signaling mechanisms downstream of Sema-3A binding. FARP2: FERM, RhoGEF and pleckstrin domain protein-2, RasGAP: plexin's latent Ras GTPase activating protein (the figure was adapted from [12]) plexin ligand, exacerbated the cytokine storm caused by TLR agonists and bacterial sepsis [11]. TLR engagement induced Sema-3A expression and thereby completed an autocrine loop. Their findings show the novel intersection of the plexin-A4 and TLR pathways through the intracellular activation of Rac1, JNK, and NF- $\mathrm{kB}$. This intersection results in chromosomal changes at the promoters of pro-inflammatory genes, followed by a pan-cytokine inflammatory response in response to TLR stimulation and bacterial challenge. Typically, inflamed tissues are associated with an elevated level of reactive oxygen species. Reactive oxygen species are generated during the respiratory burst of immune cells and are important factors in defense. Additionally, reactive species are now known to trigger oxidative/nitrosative modifications of biomolecules. The findings expand the role of plexins to TLR signaling and suggest plexin-A4 and Sema-3A as new intervention targets for treating sepsis. Similarly, the observations reported by Mohamed et al. indicate that diabetes increases Sema-3A expression, although probably not quite as fast as sepsis [8].

The answer may require further elucidation of Sema-3A signaling and its ligand action on neuropilin and plexin (Fig. 1). The Rho family of GTPases acts downstream of these axon-guidance receptors. They not only control proteins that remodel the cytoskeleton but also interact with many other signaling pathways. Evidence suggests that these GTPases also team up to regulate activation of the semaphorin-3A receptor itself [12]. The focus has been on axon guidance. Interactions between these signaling pathways and pathways leading to inflammation and reactive-oxygen-species generation would be of interest.

Respectfully,

Friedrich C. Luft

\section{References}

1. Roth L, Koncina E, Satkauskas S, Cremel G, Aunis D, Bagnard D (2009) The many faces of semaphorins: from development to pathology. Cell Mol Life Sci 66:649-666

2. Vadasz Z, Toubi E (2014) Semaphorins: their dual role in regulating immune-mediated diseases. Clin Rev Allergy Immunol 47:17-25

3. Reidy K, Tufro A (2011) Semaphorins in kidney development and disease: modulators of ureteric bud branching, vascular morphogenesis, and podocyte-endothelial crosstalk. Pediatr Nephrol 26:14071412

4. Ranganathan P, Jayakumar C, Mohamed R, Weintraub NL, Ramesh G (2014) Semaphorin 3A inactivation suppresses ischemiareperfusion-induced inflammation and acute kidney injury. Am J Physiol Renal Physiol 307:F183-F194

5. Jayakumar C, Ranganathan P, Devarajan P, Krawczeski CD, Looney S, Ramesh G (2013) Semaphorin 3A is a new early diagnostic biomarker of experimental and pediatric acute kidney injury. PLoS One 8:e58446

6. Doi K, Noiri E, Nangaku M, Yahagi N, Jayakumar C, Ramesh G (2014) Repulsive guidance cue semaphorin 3A in urine predicts the 
progression of acute kidney injury in adult patients from a mixed intensive care unit. Nephrol Dial Transplant: Off Publ Eur Dial Transplant Assoc - Eur Renal Assoc 29:73-80

7. Viazzi F, Ramesh G, Jayakumar C, Leoncini G, Garneri D, Pontremoli R (2014) Increased urine semaphorin-3A is associated with renal damage in hypertensive patients with chronic kidney disease: a nested case-control study. J Nephrol. doi:10.1007/ s40620-014-0097-5

8. Mohamed R, Ranganathan P, Jayakumar C, Nauta FL, Gansevoort RT, Weintraub NL, Brands M, Ramesh G (2014) Urinary semaphorin $3 \mathrm{~A}$ correlates with diabetic proteinuria and mediates diabetic nephropathy and associated inflammation in mice. J Mol Med (this issue). doi:10.1007/s00109-014-1209-3
9. Cerani A, Tetreault N, Menard C, Lapalme E, Patel C, Sitaras N, Beaudoin F, Leboeuf D, De Guire V, Binet F et al (2013) Neuron-derived semaphorin $3 \mathrm{~A}$ is an early inducer of vascular permeability in diabetic retinopathy via neuropilin-1. Cell Metab 18:505-518

10. Smith LE, Wesolowski E, McLellan A, Kostyk SK, D'Amato R, Sullivan R, D'Amore PA (1994) Oxygen-induced retinopathy in the mouse. Invest Ophthalmol Vis Sci 35:101-111

11. Wen H, Lei Y, Eun SY, Ting JP (2010) Plexin-A4-semaphorin 3A signaling is required for toll-like receptor- and sepsis-induced cytokine storm. J Exp Med 207:2943-2957

12. Garrity PA (2005) Tinker to Evers to Chance: semaphorin signaling takes teamwork. Nat Neurosci 8:1635-1636 